\title{
Independent null steering by decoupling complex weights
}

\author{
Z. U. Khan ${ }^{1 \mathrm{a})}$, A. Naveed ${ }^{2}$, I. M. Qureshi ${ }^{3}$, and Fawad Zaman ${ }^{1}$ \\ ${ }^{1}$ Department of Electronic Engineering, IIU, H-10, Islamabad, Pakistan \\ ${ }^{2}$ School of Engineering \& Applied Sciences (SEAS) Isra University, \\ Islamabad Campus, Pakistan \\ ${ }^{3}$ Department of Electrical Engineering Air University Islamabad, Pakistan \\ a) zafarullah.phdee13@iiu.edu.pk
}

\begin{abstract}
In this paper, we propose a structure for independent null steering by decoupling complex weights. It has the advantage of independent steering of $(N-1)$ nulls for a linear array having $N$ elements and the complex weights are decoupled such that each weight corresponds to a particular null. For this purpose, the position of each zero of the array factor on the unit circle in the complex plane is controlled by the corresponding weight in the structure. It means that if a single jammer changes its position, only a single corresponding complex weight has to be recalculated, which reduces computational complexity.
\end{abstract}

Keywords: null steering, linear array, array factor, progressive phase Classification: Science and engineering for electronics

\section{References}

[1] H. Krim and M. Viberg, "Two decades of array signal processing research," IEEE Signal Process. Mag., pp. 67-94, July 1996.

[2] J. A. Hejres, A. Peng, and J. Hijres, "Fast Method for Sidelobe Nulling in a Partially Adaptive Linear Array Using the Elements Positions," IEEE Antennas Wireless propag. lett., vol. 6, pp. 332-335, 2007.

[3] J. Hejres, "Array pattern nulling using the elevations of selected elements of phased antenna arrays," Proc. IEEE Int. Symp. AP-S, 2005, vol. 2B, pp. 68-71, June 2005.

[4] H. M. Ibrahim, "Null Steering by Real-Weight Control - A Method of Decoupling the Weights," IEEE Trans. Antennas Propag., vol. 39, no. 11, Nov. 1991.

[5] J. A. Hejres, "Null Steering in Phased Arrays by Controlling the Position of Selected Elements," IEEE Trans. Antennas Propag., vol. 52, no. 11. pp. 2891-2895, Nov. 2004.

[6] T. B. Vu, "Simultaneous nulling in sum and difference pattern by amplitude control," IEEE Trans. Antennas Propag., vol. AP-34, pp. 214-218, Feb. 1986. 


\section{Introduction}

Null steering for adaptive antenna system has been a subject of intensive interest in past [1] and has its importance even to date [2]. It has applications in the fields of radar and wireless communications. There are traditional techniques to find an appropriate set of complex weights for the elements of a linear array to form the main beam towards the desired signal direction and nulls along interferences. There are also alternative approaches like position perturbation or the element elevation, for null steering but these approaches are slow for large arrays because the computational time increases to find new position perturbation or elevation for these arrays. Fast algorithms are being developed for the alternative techniques by controlling the position of selected elements, [2,3] and [5] i.e. the movement of all the elements is avoided. The setback with these techniques is that they require servo motors to move every element in the array. Like alternative approaches, the traditional techniques are also slow for large arrays because if the position of a single null is required to change due to the movement of a jammer, the whole set of complex weights has to be recalculated. Efforts have been made to search more effective techniques. A technique presented in [6] reduces the computation time up to significant level. Independent null steering method is proposed in [4] where the computation is required only for the desired position of corresponding null and computation time is reduced drastically. But in [4] and [6], the number of steerable nulls reduce to less than or equal to half the number of total elements.

The proposed scheme presents a method to steer all possible nulls independently. The method utilizes an array of complex weights and a set of two-input summers. Each stage of the array is responsible to steer a particular null and has a set of identical weights i.e. each weight in a stage has same value. If the position of a particular null is required to change, the set of identical weights in the corresponding stage is changed.

\section{Background}

Consider a uniform linear array of $N$ elements with constant element spacing $d$ and progressive phase $\alpha$ as shown in fig. 1. Taking element-1 as reference element and let $\theta$ be the angle of arrival.

Let the array factor for this array be denoted as $A F^{\prime}$ and is given by

$$
A F^{\prime}=\sum_{i=1}^{N} e^{j(i-1) \psi}
$$

where $\psi=\alpha+(2 \pi / \lambda) d \cos \theta$

Let $e^{j \psi}=z$

$$
A F^{\prime}=\sum_{i=1}^{N} z^{(i-1)}=1+z+\ldots+z^{(N-1)}
$$

The polynomial in $(2)$ is of $(N-1)$ degree with $(N-1)$ roots. It can be 


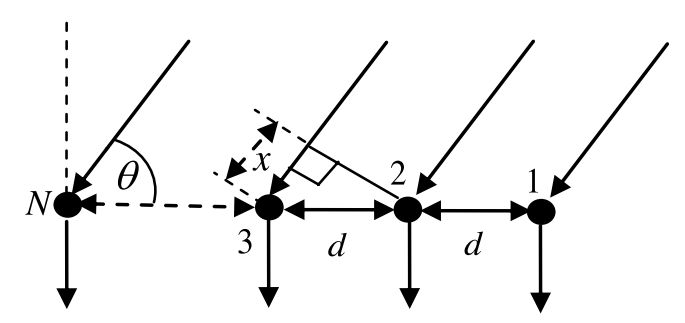

Fig. 1. Uniform linear array of $N$ elements

written as

$$
A F^{\prime}=\sum_{i=1}^{N} z^{(i-1)}=1+z+\ldots+z^{(N-1)}
$$

This means, there exist zeros at $z_{1}^{\prime}, z_{2}^{\prime} \ldots z_{N-1}^{\prime}$ and these zeros correspond to nulls in the $A F^{\prime}$ along fixed directions $\theta_{1}^{\prime}, \theta_{2}^{\prime} \ldots \theta_{N-1}^{\prime}$. In order to steer nulls along arbitrary directions $\theta_{1}, \theta_{2} \ldots \theta_{N-1}$, the array factor should be modified as

$$
A F=A_{0}+A_{1} z+\ldots+A_{N-1} z^{(N-1)}
$$

This array factor is also a polynomial of degree $(N-1)$ and has $(N-1)$ adjustable roots dependent on the selection of suitable coefficients $A_{0}, A_{1}, \ldots$, $A_{N-1}$. In factorized form (4) becomes

$$
A F=\left(z-z_{1}\right)\left(z-z_{2}\right) \ldots\left(z-z_{N-1}\right)
$$

where $z_{1}, z_{2}, \ldots z_{N-1}$ are chosen so as to steer the nulls along required directions.

The progressive phase shift $\alpha$ is given by

$$
\alpha=-(2 \pi / \lambda) d \cos \theta_{s}
$$

\section{Proposed structure for independent null steering}

First, proposed structure for a uniform linear array of 5 elements is discussed and then its results are generalized for an $\mathrm{N}$-element array.

It can be seen from fig. 2 that each summer has two inputs. In first stage, first input of each summer is multiplied by $\left(-z_{1}\right)$. This $z_{1}$ corresponds to the position of first null in the array factor.

Each output of first stage is denoted by $y_{1, j}$, for $j=1, \ldots, N-1$. So, $y_{1,1}=z-z_{1}, y_{1,2}=z\left(z-z_{1}\right), y_{1,3}=z^{2}\left(z-z_{1}\right), y_{1,4}=z^{3}\left(z-z_{1}\right)$

The general formula for first stage outputs of an $N$-element array is given by

$$
y_{1, j}=z^{j-1}\left(z-z_{1}\right) \text { for } j=1, \ldots, N-1
$$

Similarly, each output of 2 nd stage is denoted by $y_{2, j}$ for $j=1, \ldots, N-2$

So, $y_{2,1}=\left(z-z_{1}\right)\left(z-z_{2}\right), y_{2,2}=z\left(z-z_{1}\right)\left(z-z_{2}\right), y_{2,3}=z^{2}\left(z-z_{1}\right)\left(z-z_{2}\right)$. The general formula for 2 nd stage outputs of an $\mathrm{N}$-element can be expressed as

$$
y_{2, j}=z^{j-1}\left(z-z_{1}\right)\left(z-z_{2}\right) \text { for } j=1, \ldots, N-2
$$




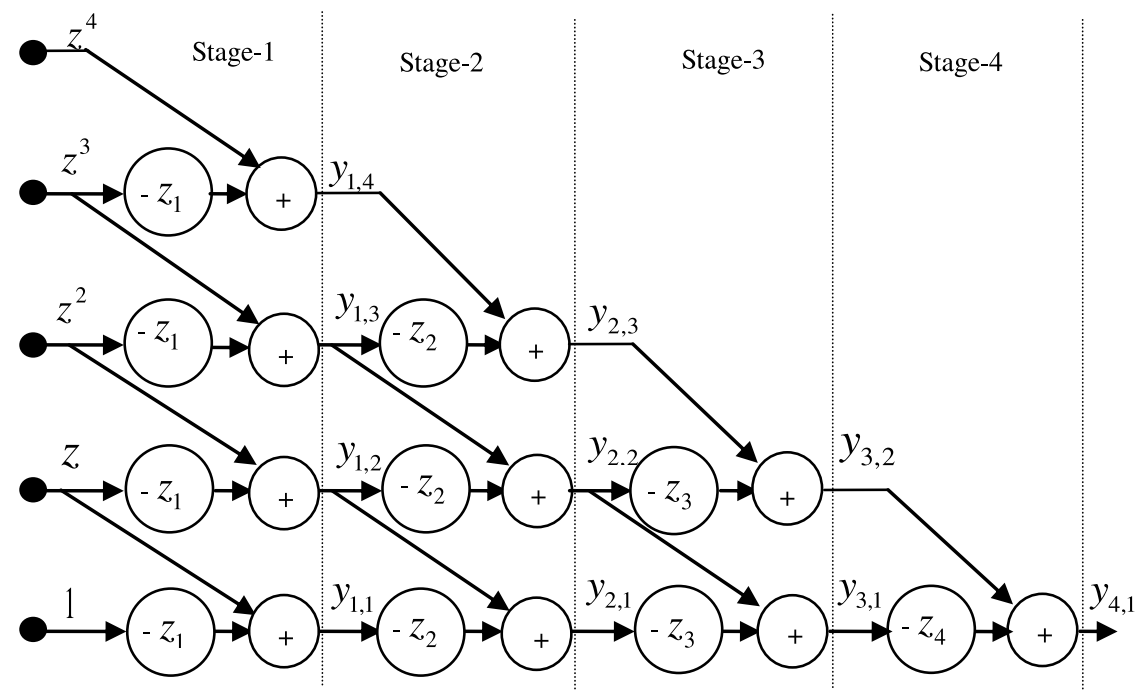

Fig. 2. Proposed structure for uniform linear array of 5 elements

Third stage outputs of fig. 2 can be expressed as

$$
y_{3,1}=\left(z-z_{1}\right)\left(z-z_{2}\right)\left(z-z_{3}\right), \quad y_{3,2}=z\left(z-z_{1}\right)\left(z-z_{2}\right)\left(z-z_{3}\right)
$$

The general formula for this stage outputs with $N$ elements is given as

$$
y_{3, j}=z^{j-1}\left(z-z_{1}\right)\left(z-z_{2}\right)\left(z-z_{3}\right) \text { for } j=1, \ldots, N-3
$$

Finally, we consider the 4 th stage output for fig. 2

$$
y_{4,1}=y_{3,2}-z_{4} y_{3,1}=\left(z-z_{1}\right)\left(z-z_{2}\right)\left(z-z_{3}\right)\left(z-z_{4}\right)
$$

Now we can write a general formula for output of each stage for an array having $N$ elements. This array will have $(N-1)$ stages.

$$
y_{i, j}=z^{j-1}\left(z-z_{1}\right)\left(z-z_{2}\right) \ldots\left(z-z_{k}\right)
$$

In the above relation, $i=1, \ldots N-1, j=1, \ldots, N-i, k=1, \ldots i$

The final output for this array will be

$$
y_{N-1,1}=\left(z-z_{1}\right)\left(z-z_{2}\right) \ldots\left(z-z_{N-1}\right)
$$

The relation between interference direction $\theta_{i}$ and corresponding $z_{i}$ is given as

$$
z_{i}=\exp \left(j\left(\alpha+(2 \pi / \lambda) d \cos \theta_{i}\right)\right)
$$

\section{Simulation results}

For simulation a uniform linear array of 5 elements with inter element spacing $\lambda / 2$ is considered here. This arrangement can steer four nulls independently. Desired signal is taken along $\theta_{s}=100^{\circ}$. Progressive phase shift $\alpha$ related to $\theta_{s}=100^{\circ}$ is $-\pi \cos \theta_{s}$. The direction $\theta_{i}$ of interferences for four jammers are taken as: $\theta_{1}=50^{\circ}, \theta_{2}=80^{\circ}, \theta_{3}=120^{\circ}, \theta_{4}=160^{\circ}$, and the corresponding 
weights are, $z_{1}=-0.8383+0.5452 \mathrm{i}, z_{2}=0.4615+0.8871 \mathrm{i}, z_{3}=0.5189-$ $0.8549 \mathrm{i}$, and $z_{4}=-0.7418-0.6706 \mathrm{i}$ respectively.

Jammer 1 moves from $\theta_{1}=50^{\circ}$ to $\theta_{1}=55^{\circ}$ and the corresponding weight is $z_{1}=-0.7009+0.7132 \mathrm{i}$. This independent movement of null is shown in fig. 3a. Jammer 2 moves from $\theta_{2}=80^{\circ}$ to $\theta_{2}=75^{\circ}$ and the corresponding weight is $z_{2}=0.2106+0.9776 \mathrm{i}$. This independent movement of null is shown in fig. $3 \mathrm{~b}$. Jammer 3 moves from $\theta_{3}=120^{\circ}$ to $\theta_{3}=125^{\circ}$ and the corresponding weight is $z_{3}=0.3092-0.9510 \mathrm{i}$. This independent movement of null is shown in fig. 3c. Jammer 4 moves from $\theta_{4}=160^{\circ}$ to $\theta_{4}=155^{\circ}$ and the corresponding weight is $z_{4}=-0.6676-0.7446 \mathrm{i}$. This independent movement of null is shown in fig. 3d. In the four cases mentioned above, the main beam is always directed along desired direction $\theta_{s}=100^{\circ}$ and the directions of jammers do not affect it. It can be steered by changing the progressive phase shift $\alpha$. However, if there is a change in the direction of desired signal, all the weights are to be recalculated according to (12).
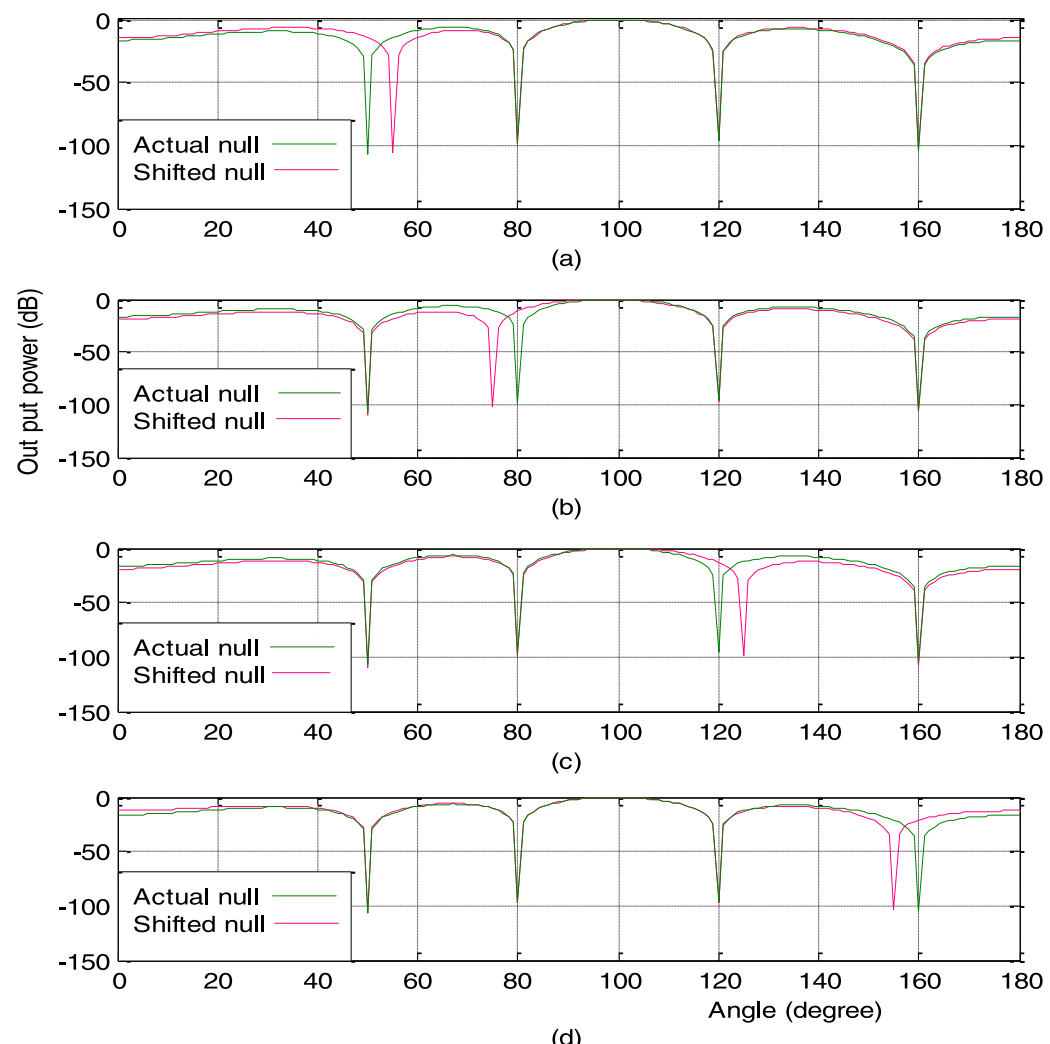

(d)

Fig. 3. (a) Independent movement of null from $50^{\circ}$ to $55^{\circ}$,

(b) Independent movement of null from $80^{\circ}$ to $75^{\circ}$,

(c) Independent movement of null from $120^{\circ}$ to $125^{\circ}$, (d) Independent movement of null from $160^{\circ}$ to $155^{\circ}$

\section{Conclusion}

The method of independent null steering using complex weights is offered. The weights are decoupled such that each null can be steered with a corre- 
sponding weight value. Only corresponding weight value is required to change if the change in the direction of any interference takes place. The advantage of complex weight is that the number of steerable nulls is maintained to their maximum value. The price for this method is that the number of complex weights is high. A uniform linear array of $N$ elements can steer $(N-1)$ nulls using $N(N-1) / 2$ complex weights. These weights have $(N-1)$ set of values. If the number of jammers to be suppressed is small compared to the number of steer able nulls, the remaining nulls can be used to control the sidelobes. For example, the pattern of fig. 3 can be improved if there are three jammers at $\theta_{1}=50^{\circ}, \theta_{2}=80^{\circ}, \theta_{3}=160^{\circ}$, and the fourth surplus null is placed at $130^{\circ}$ instead of $120^{\circ}$. 